\title{
Analysis of a spatial Lotka-Volterra model with a finite range predator-prey interaction
}

\author{
E. Brigatti ${ }^{\dagger}$, M. Núñez-López ${ }^{ \pm}$and M. Oliva ${ }^{\ddagger}$ \\ $\dagger$ Instituto de Cincias Exatas, Universidade Federal Fluminense - Rua E. H. Figueira, 783, RJ, Brasil \\ Instituto de Fúsica, Universidade Federal Fluminense - Campus da Praia Vermelha, 24210-340, Niterói, RJ, Brasil \\ \pm Instituto Mexicano del Petróleo - Eje Central Lázaro Cárdenas Norte 152, Gustavo A. Madero, 07730 DF, México \\ $\ddagger$ Facultad de Física, Universidad de La Habana - Ave. Universidad y Ronda, Vedado, 10400, Havana, Cuba
}

\begin{abstract}
We perform an analysis of a recent spatial version of the classical Lotka-Volterra model, where a finite scale controls individuals' interaction. We study the behavior of the predator-prey dynamics in physical spaces higher than one, showing how spatial patterns can emerge for some values of the interaction range and of the diffusion parameter.
\end{abstract}

PACS numbers: 87.23.Cc, 05.10.-a, 05.45.-a

\section{INTRODUCTION}

Reaction-diffusion theory plays a very important role in the study of pattern formation in biology, an aspect of populations dynamics which has gained importance since some Moran's seminal works [1,2]. More recently, theoretical ecology studies focused on the dynamical mechanisms which can be considered responsible for producing such structures. Their principal aim is to determine the local rules which cause the emergence of macroscopic patterns [7]. At present, a central issue is to determine whether the predator-prey interactions can be considered among these mechanisms. Actually, spatial correlations between predators and preys populations have been observed in real systems such as predatory beetles and larval flies as their preys [3]. This research shows that these larval flies appear to gain local clustering as beetle abundance approaches a carrying capacity with the prey population.

A traditional approach for describing these systems is based on spatial Lotka-Volterra type models. This description is able to generate spatial structures of the Turing type, where the instabilities are directly driven by mechanisms connected with the process of diffusion [10]. These works are inspired to the classical ideas introduced by Turing, who showed that diffusion can destabilize the homogeneous equilibrium solution of a reaction-diffusion system [9]. This topic has recently received some attention, in particular in relation to the effects of stochasticity. For example, in [4] it is shown that Turing-like patterns can indeed emerge beyond the parameter region predicted by the conventional Turing theory. This phenomenon is due to the effect of finite size corrections on the mean-field idealized dynamics. Also demographic noise can induce persistent spatial pattern formation and temporal oscillations [5]. Specifically, demographic noise greatly enlarges the region of parameters space where pattern formation occurs, in relation to the prediction of the mean-field theory. Finally, Scott et. al. [6] introduce an analytic method to describe stochastic spatially varying systems governed by reaction-transport master equation.

More recently, a different mechanism capable of generating spatial patterns has been explored [13]. It is based on the introduction of a probability of interaction (predation) which becomes a function of the distance between individuals. This description presents two interesting points. First, it is not subject to the strong constraints which limit the emergence of spatial inhomogeneities in the traditional approach [14]. Second, it is based on a simple ingredient which has a deep ecological motivation. In fact, it has became evident that predation strongly depend on probability of encounter [8] which must take into account predators shifts in response to prey movements. Moreover, territoriality is another fundamental aspect in the ecology of many predatory animals such as wolves, lions, hyenas, African wild dogs and badgers. In this case an immediate question arises as to how the predation is strongly influenced by a land which is divided up into predator territories [10]. These elements result in a spatial and temporal variation in the predation risk [12]. A possible description of one aspect of this spatial dependence can be obtained considering that the probability at which a consumer meets a prey should be dependent on the relative distance between them [15]. The introduction of a spatial scale of interaction has been widely applied to model competition in community ecology [15] and more recently it was also used in studies of evolutionary theory [16].

In the following, we present a detailed analysis of this approach for an implementation of a predator-prey system in a two dimensional physical space, the most relevant for describing real ecosystems.

\section{THE MEAN-FIELD MODEL}

We start with the mean-field version of the model. This description is characterized by a couple of equations, one for the preys $N(\bar{x}, t)$ and other for the predators $P(\bar{x}, t)$. They describe diffusion in real space and the strength of the interaction in the nonlinear term is 
a function of the density of individuals in the proximity [17]. The reason for introducing a spatial scale for the interaction can be clearer understood considering that, based on the reported ecological motivations, we want to describe a probability of reproduction/death not constant but dependent on the number of predators/preys in the surrounding. In fact, in a region more populated by preys, predators should have a better chance of reproduction. Conversely, in a region more populated by predators, preys should have a greater chance of dying. This nonlocality appears as an integral term which takes into account the local density of population. It is important to point out that this effect is not related to the individuals' velocity, controlled by the diffusion coefficients, but to the memory which individuals maintain in relation to a specific territory. Territoriality is a fundamental aspect in the ecology of many predatory animals [10]. For this reason, as the range of interaction is not simply linked to the relative motion between predators and preys, it does not depend on a single interaction scale.

Considering a two dimensional physical space the equations which describe our system are the following:

$$
\begin{aligned}
\frac{\partial N(\bar{x}, t)}{\partial t}= & D_{N} \frac{\partial^{2} N(\bar{x}, t)}{\partial \bar{x}^{2}}+r N(\bar{x}, t) \\
& -\alpha N(\bar{x}, t) \int_{\left|\bar{x}^{\prime}-\bar{x}\right|<R_{1}} P\left(\bar{x}^{\prime}, t\right) d \bar{x}^{\prime} \\
\frac{\partial P(\bar{x}, t)}{\partial t}= & D_{P} \frac{\partial^{2} P(\bar{x}, t)}{\partial \bar{x}^{2}}-m P(\bar{x}, t) \\
& +\beta P(\bar{x}, t) \int_{\left|\bar{x}^{\prime}-\bar{x}\right|<R_{2}} N\left(\bar{x}^{\prime}, t\right) d \bar{x}^{\prime} .
\end{aligned}
$$

where $\bar{x}=(x, y)$ is the position of predators or preys. Predators consume the preys with an intrinsic rate $\alpha$ and reproduce with rate $\beta, r$ is the prey's growth rate and predators are assumed to spontaneously die with rate $m$. $D_{N}$ and $D_{P}$ are the diffusion coefficients of preys and predators, respectively.

A microscopic version of these equations is introduced in the next section. There, the integral terms correspond to counting the number of preys/predators which are at a shorter distance than $R_{i}$ from the considered predator/prey.

We would like to point out that a rigorous derivation, which would show that the continuum field equations introduced here are totally analogous to the discrete model presented in the next section, can be obtained by using Fock space techniques after making some usual assumption and approximation $[17,18]$.

To perform this passage between discrete model and mean field description the microscopic model should be defined on a lattice. The lattice model will be equivalent to the off-lattice one in the limit of small lattice size. For this reason the off-lattice model present in the next section should be modified to fit into a grid. If we let the grid spacing to become negligibly small we recover the continuum limit. With the aim of preserving the non-locality of our macroscopic model, in which the interaction range is finite, this operation of limit should be made carefully. In fact, only the grid size, but not the interaction lengths $R_{i}$ should vanish. This is obtained fixing $R_{i}$ to a finite value when going to the continuum.

Analogous models, but not characterized by a finite scale of interaction, appeared recently in the literature. Spatio-temporal patterns can emerge sustained by the stochastic component of the dynamics. In this case, the demographic fluctuations, directly reproduced in the individual based description level, are the responsible for pattern formation $[5,19]$.

The one dimensional case was deeply analyzed in the reference [13]. In the following we will present some results related to the two-dimensional physical space, and, for theoretical interest, to n-dimensional spaces.

Also in the two dimensional space, an absorbing phase $N(\bar{x}, t)=P(\bar{x}, t)=0$ and a survival phase $\bar{N}(\bar{x}, t)=$ $\frac{m}{\pi \beta R_{2}^{2}} ; \bar{P}(\bar{x}, t)=\frac{r}{\pi \alpha R_{1}^{2}}$ exist and they correspond to spatially homogeneous, stationary solutions.

The analysis for detecting the presence of solutions with spatial structure is based on the idea of perturbing the survival phase stationary solution $\bar{N}(\bar{x}, t)$ and $\bar{P}(\bar{x}, t)$. This is obtained introducing small harmonic perturbations [10,17]: $N(\bar{x}, t)=\bar{N}+A_{N} \exp [\lambda t+i \bar{k} \cdot \bar{x}]$; $P(\bar{x}, t)=\bar{P}+A_{P} \exp [\lambda t+i \bar{k} \cdot \bar{x}]$, where $\bar{k}=\left(k_{x}, k_{y}\right)$ is a two-dimensional wave vector of modulus $|\bar{k}|=k$. The simple harmonic form of these perturbations reduces equations 1 to a linear system. The solution of this system generates some constraints on the values which $\lambda$ and $k$ can assume. This dispersion relation can be written in the following form:

$$
\begin{aligned}
\lambda(k)= & -\frac{k^{2}}{2}\left(D_{N}+D_{P}\right) \\
& \pm \sqrt{\frac{k^{4}}{4}\left(D_{N}-D_{P}\right)^{2}-4 r m \frac{J_{1}\left(k R_{1}\right) J_{1}\left(k R_{2}\right)}{k^{2} R_{1} R_{2}}},
\end{aligned}
$$

where $J_{1}$ is the first-order Bessel function.

If, for some $k, \operatorname{Re}[\lambda(k)]>0$ spatial patterns can emerge. It follows that $R_{1} \neq R_{2}$ is a necessary condition. Moreover, because for $D_{N}=D_{P}=0$ the condition is always satisfied, it is evident that the instability is driven by the range of the interaction and is independent of the diffusion process.

If we consider the simpler situation with $D_{N}=D_{P}=$ $D$ and $R_{1}=2 R_{2}=2 R$, and we introduce the rescaled variables $K=k R$ and $\widehat{\lambda}=\lambda \frac{R^{2}}{D}$, for $\mu=\sqrt{r m} R^{2} / D$ equation 2 reduces to:

$$
\widehat{\lambda}(K)=-K^{2}+\frac{\mu}{K} \sqrt{-J_{1}(K) J_{1}(2 K)} .
$$

The edge of spatial patterns emergence corresponds to the values of the parameters for which the maximum $K_{m}$ of $\widehat{\lambda}(K)$ becomes positive. For the original variables this happens if:

$$
\frac{\sqrt{r m} R^{2}}{D} \gtrsim 22.4228 \quad \text { and } \quad k_{m} \approx \frac{2.19535}{R} .
$$

Figure 1 shows $\widehat{\lambda}(K)$ for different $\mu$ values. 


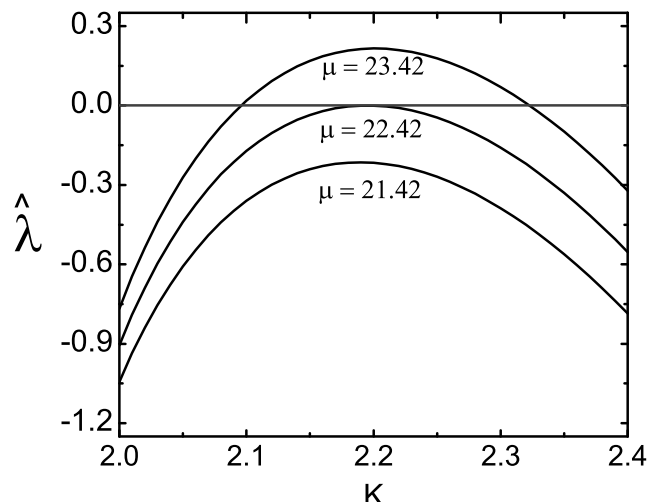

FIG. 1. Dispersion relation $\widehat{\lambda}(K)$ for different values of the parameters $\left(\mu=\sqrt{r m} R^{2} / D\right)$.

In the following we turn our attention to the model implemented in a $n$-dimensional space, with $n>2$. In this case, the corresponding equations are obviously obtained generalizing the Laplacian terms and the integration domains. Performing a calculation analogous to the 2-dimensional case, we arrive at the following dispersion relation:

$$
\begin{aligned}
\lambda(k) & =-\frac{k^{2}}{2}\left(D_{N}+D_{P}\right) \\
& \pm \sqrt{\frac{k^{4}}{4}\left(D_{N}-D_{P}\right)^{2}-r m 2^{n} \Gamma\left(1+\frac{n}{2}\right)^{2} \frac{J_{n / 2}\left(k R_{1}\right) J_{n / 2}\left(k R_{2}\right)}{\left(k R_{1}\right)^{n / 2}\left(k R_{2}\right)^{n / 2}}},
\end{aligned}
$$

where $n$ is the space dimension. Introducing the variables $K=k R_{2}, \rho=\frac{R_{1}}{R_{2}}$ and $\mu=R_{1} R_{2} \sqrt{\frac{r m}{D_{N} D_{P}}}$, spatial patterns can emerge if the following condition is satisfied:

$$
\frac{\rho^{2} K^{4}}{\mu^{2}}+2^{n} \Gamma\left(1+\frac{n}{2}\right)^{2} \frac{J_{n / 2}(K) J_{n / 2}(\rho K)}{\rho^{n / 2} K^{n}}<0 .
$$

As for the two dimensional situation, a critical value $\mu_{c}$ exists for which, if $\mu>\mu_{c}$ spatial patterns emerge. In Figure 2 we show the $\mu_{c}^{-1}$ values as a function of the ratio $\rho=\frac{R_{1}}{R_{2}}$ for different dimensions. The value of $\mu_{c}^{-1}$ increases as the parameter $\rho$ increases. This means that a stronger difference between $R_{1}$ and $R_{2}$ leads to easier cluster formation. Moreover, it is clear that for $\rho>1$ clustered distributions can appear also for a physical dimension greater than 2. In higher dimensions cluster formation becomes more difficult but it is viable. It is important to remember that even the case of a 3dimensional space is not a common ecosystem. Obviously it does not exist for terrestrial individuals and even marine species tend to remain within a layer of small thickness compared to their horizontal movements. Anyway, our results for dimension higher than 2 have a theoretical interest. In fact, qualitative differences depending on the space dimension are a common feature in systems with diffusive phenomena and clusters formation (see for example [20]).

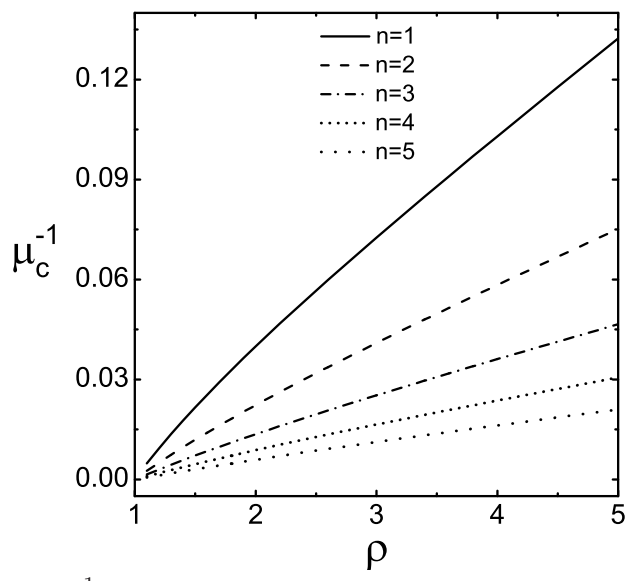

FIG. 2. $\mu_{c}^{-1}$ values in dependence of $\rho$ for different physical dimensions $n$.

\section{THE MICROSCOPIC DISCRETE MODEL}

In the following we introduce a microscopic discrete stochastic formulation of the mean field model. The importance of comparing the results of the mean-field description with an individual based one is due to the possibility of introducing demographic fluctuations. These fluctuations result in the appearance of an intrinsic stochasticity which, in principle, is always present in real phenomena. Moreover, this technique can describe the occurrence of threshold effects, generated by the discrete nature of individuals. These effects are not present in the mean field description where every small amount of the density of population is acceptable, even if unrealistically small [21].

We implement our individual based model on the square $[0,1] \times[0,1]$, with periodic boundary conditions. Simulations start with an initial population of $P_{0}$ predators and $N_{0}$ preys, randomly located. A time step of our simulation corresponds to carrying out the following processes: 1) diffusion, where a predator, or a prey, is randomly selected and moves some distance, in a random direction, chosen from a Gaussian distribution of standard deviation $\sigma$. 2) predator reproduction, with rate $\beta N_{R_{2}}^{\bar{x}}$, where $N_{R_{2}}^{\bar{x}}$ is the number of preys which are at a shorter distance than $R_{2}$ from the predator at position $\bar{x}$. 3) predator death, with probability $m$. 4) prey reproduction, with probability $r$. 5) prey death, with rate $\alpha P_{R_{1}}^{\bar{y}}$, where $P_{R_{1}}^{\bar{y}}$ is the number of predators which are at a shorter distance than $R_{1}$ from the prey at position $\bar{y}$. All the newborns maintain the same location as the parents. We evaluate $N_{R_{2}}^{\bar{x}}$ and $P_{R_{1}}^{\bar{y}}$ using periodic boundary conditions. These processes are executed sequentially by the whole population. In fact, for each action, an individual of each population (predator or prey) is randomly selected and this operation is repeated for a number of times equal to the size of the corresponding population.

It is important to point out that if we measure time in units of the simulation time step, the coefficient $D$ of 
equation 1 is related to the discrete model through the relation $D=\sigma^{2} / 2$. Birth and death probabilities are the same in the continuous and in the discrete model.

Analyzing the data generated throughout these simulations we are able to explore the temporal and spacial behavior of our system. The temporal dynamics of our simulations is strongly dependent on the form of the spatial organization of the populations. The temporal evolution of the simulations characterized by homogeneous spatial distributions can be easily understood considering a classical Lotka-Volterra model. In fact, if we start with an initial population of $P_{0}=\frac{r}{\pi \alpha R_{1}^{2}}$ predators and $N_{0}=\frac{m}{\pi \beta R_{2}^{2}}$ preys, corresponding to the two stationary solutions of the mean field model, inevitable fluctuations tend to push the system away from the trivial survival phase and induce irregular population oscillations that almost resemble the deterministic cycles of the classical Lotka-Volterra model. This behaviour has been described also in other analogous stochastic models $[22,18]$. We must remember that an important outcome of the introduction of intrinsic stochasticity is the possibility of predators' extinction. This fact, combined with the possibility of extremely rapid increase in the number of preys, force to a careful choice of the initial conditions and parameters of our simulational runs. This is necessary to obtain controlled irregular oscillations, which swing in a rather erratic fashion around an average value (see Figure 3). Otherwise, the explosion or extinction of population do not allow to carry out sufficiently long standing simulations.

The situation changes when the system is evolving in the spatial clustered regime. In fact, for some values of the parameters, the survival phase of the homogeneous distribution is different from the clustered one. For example, starting with $P_{0}=\frac{r}{\pi \alpha R_{1}^{2}}$ predators and $N_{0}=\frac{m}{\pi \beta R_{2}^{2}}$ preys, after a rapid transient, when spatial structures emerge, the oscillations organise around a new survival phase. In fact, the difference between $P_{R_{1}}^{\bar{y}}$ and $N_{R_{2}}^{\bar{x}}$ becomes irrelevant and the values of $\alpha$ and $\beta$ determine the ratio in the new population equilibrium (see Figure 4). This behaviour can be easily understood considering that the dimension of the bulk of the clusters is generally smaller than $R_{i}$, as shown at the end of this section. We can also remark that the more the spatial solution is marked by clustering (lower $R$ and $D$ values), the more the irregularities of the temporal oscillations increase. It is interesting to point out a recent model which shows analogous time oscillations in predators and preys number associated with a spatial structure. These oscillations are due to stochastic fluctuations about the time-independent solutions of the deterministic equations [11]. Other models describe similar oscillating behaviour [24]. Interestingly, these works identify and analytically describe a mechanism of amplification of demographic noise which can give rise to coherent oscillations. In particular, as in our model, in references $[5,19]$ these oscillations are spatiotemporal in nature.

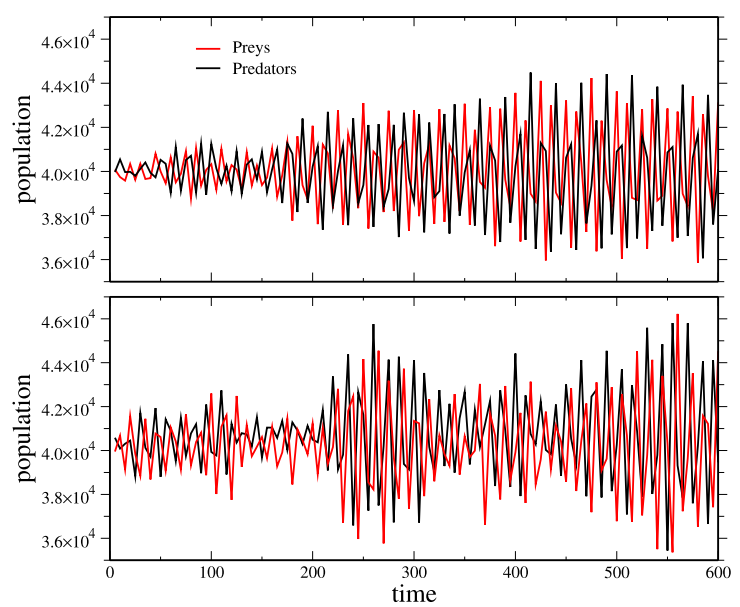

FIG. 3. Temporal evolution for populations with homogeneous spatial distributions. Top: $R_{1}=2 R_{2}=0.16$, $\sigma=0.03, r=m=0.5, \alpha=6.22 \times 10^{-4}, \beta=1.55 \times 10^{-4}$, $P_{0}=N_{0}=40000$. Bottom: $R_{1}=R_{2}=0.08, \sigma=0.008$, $r=m=0.5, \alpha=\beta=6.22 \times 10^{-4}, P_{0}=N_{0}=40000$. The two temporal evolutions are very similar. This analogous behaviour is caused by the fact that no patterns are generated in either of the two simulations: in the upper one because of the large value of $\sigma$, in the lower one because of $R 1=R 2$.

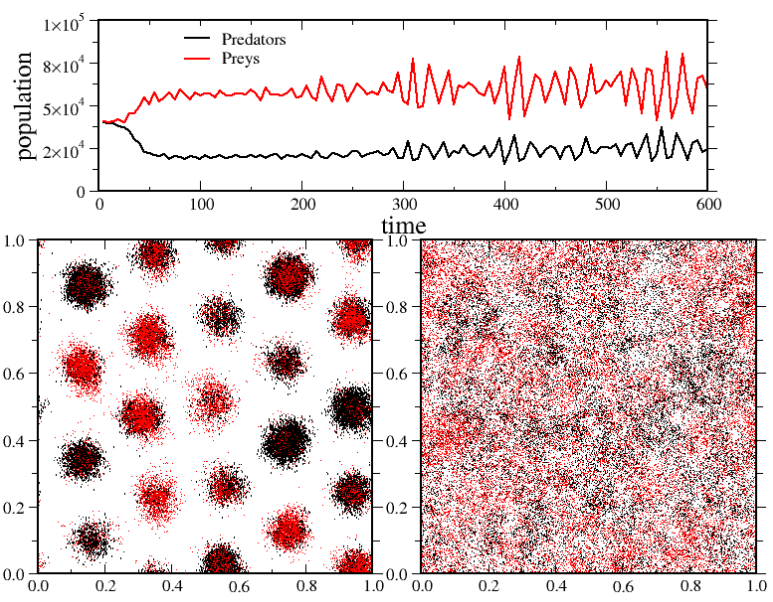

FIG. 4. Top: temporal evolution for populations with modulated spatial distributions $\left(R_{1}=2 R_{2}=0.16\right)$. Other parameters are: $\sigma=0.008, r=m=0.5$, $\alpha=6.22 \times 10^{-4}, \beta=1.55 \times 10^{-4}, P_{0}=N_{0}=40000$. Bottom: on the left, modulated spatial distribution corresponding to the simulation shown on the top of the figure. On the right, homogeneous distribution generated using the following parameters: $R_{1}=R_{2}=0.08, \sigma=0.008, r=m=0.5$, $\alpha=\beta=6.22 \times 10^{-4}, P_{0}=N_{0}=40000$.

In the following we present some results related to the spatial distributions generated by our model. As reported in Figure 4 regular patterns can be obtained only for simulations where the two ranges of interac- 
tion, $R_{1}$ and $R_{2}$, have a different value. This outcome is in agreement with the results obtained by the mean field description. The spatial patterns are characterised by a sequence of isolated colonies, generally called of spikes $[17,23]$. These spikes keep changing along the time, according to the oscillation reported in the temporal evolution of the total population (top of Figure 4). These dynamics result in periodic stationary spatial structures characterised by fluctuating clusters arranged on an hexagonal lattice. We can remark that changing the geometry of the domains of integration it is possible to generate different clusters arrangements (for example, a square domain generates clusters arranged on a square lattice).

In the following, we characterise the transition between the homogeneous spatial distribution and the inhomogeneous one (segregation transition). A proper order parameter is obtained evaluating a structure factor $S(K)$ defined as:

$$
S(K)=\left\langle\left|\sum_{j=1}^{N(\tau)} \frac{1}{N(\tau)} \exp \left[i \bar{q} \cdot \bar{x}_{j}(\tau)\right]\right|^{2}\right\rangle_{K}
$$

where the sum is carried out over all predators (or preys) $N(\tau)$ at time $\tau, \bar{x}_{j}(\tau)=\left(x_{j}(\tau), y_{j}(\tau)\right)$ is the position of the predator $j, \bar{q}=\left(q_{x}, q_{y}\right)$ is a two-dimensional wave vector and the average is a spherical average over all wave vectors of modulus $|\bar{q}|=K$. The position of the secondary local maximum of this function $\left(K_{M}\right)$ identifies the emergence of periodic structures in the spatial distribution. In fact, the transition from a homogeneous to an inhomogeneous distribution matches the jump of $K_{M}$ to higher values, corresponding to the wave number of the periodic clusters present in the space. This is clearly shown in the insets of Figure 5. The segregation transition is characterised by the passage of $K_{M}$ from small values to higher values as soon as a modulation becomes dominant $[17,23]$.

In Figure 5 we show $K_{M}$ as a function of $\sigma$. As predicted by the mean field approximation, for any value of the range of the interaction, a critical value of the diffusion coefficient exists above which no spatial structures emerge. In the figure we show some results obtained fixing the parameters $R_{1}=2 R_{2}=0.16$. For the sake of simplicity we explored the case with $R_{1}=2 R_{2}$, but similar behaviour can be obtained for other values of $\rho \neq 1$.

A similar analysis can be unfolded fixing $\sigma$ and looking for the $R$ dependence. We obtained spatial modulations of arbitrary wavelengths just tuning the parameter $R_{2}$. In Figure 6 we can see how the continuous description can reproduce quantitatively the period of the patterns which emerges from the modulated distributions of the Monte Carlo simulations. In fact, the wavenumber of these distributions are well approximated by the second relation in equation 4, which gives the fastest growing mode of the mean-field approximation. For extremely short-ranged interaction, a noisy spatially homogeneous distribution appears. This means that clusters appear for
$R_{2}>R_{c}$, where $R_{c}$ is a critical value of the interaction length for which the segregation transition takes place.

It is interesting to remark another phenomenon. Also in a two dimension implementation, independently of the values of $R$, for low $D$ values and low population density the demographic fluctuations can introduce a source of spatial correlation which can generate disordered clusters [13].

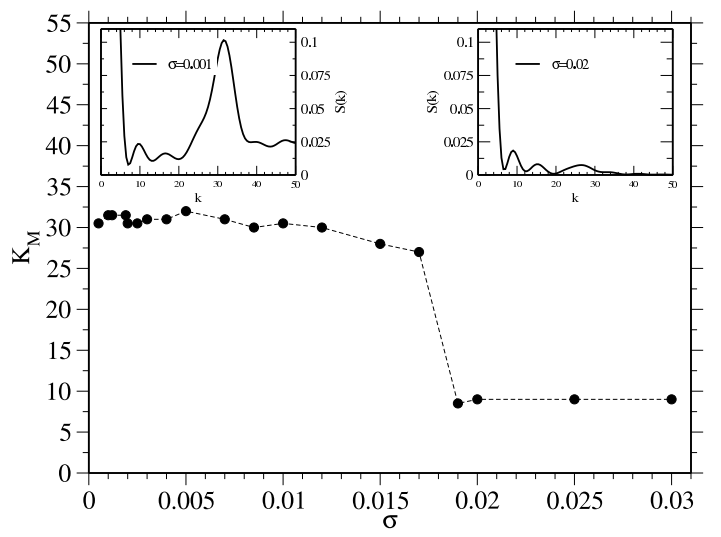

FIG. 5. $K_{M}$ as a function of $\sigma\left(R_{1}=2 R_{2}=0.16\right.$, $r=m=0.5, \alpha=6.22 \times 10^{-4}, \beta=1.55 \times 10^{-4}$, $\left.P_{0}=N_{0}=40000\right)$. For this parameters the analytical prediction gives $\sigma_{c}=0.019$, in good accordance with the data obtained from the discrete model.

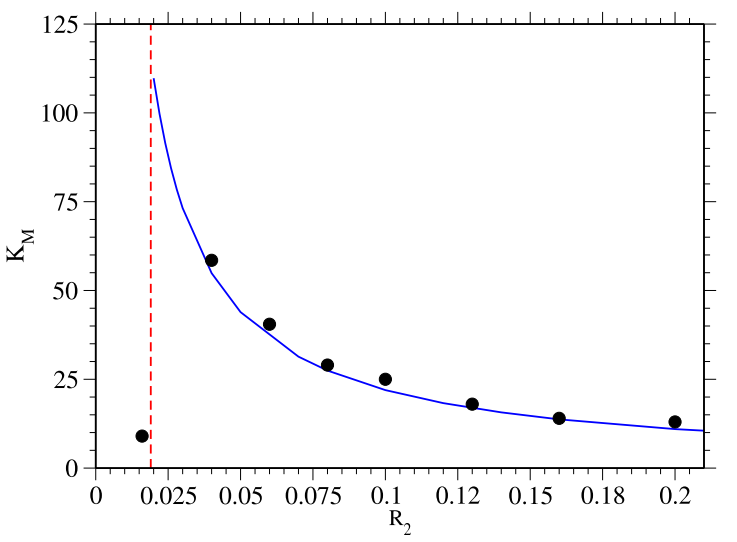

FIG. 6. $K_{M}$ as a function of $R_{2} \quad(\sigma=0.004$, $r=m=0.5, R_{1}=2 R_{2}, P_{0}=N_{0}=80000, \alpha=\left[2 \pi P_{0} R_{2}^{2}\right]^{-1}$, $\beta=0.25 \alpha$ ). Simulational data are compared with the analytical predictions given by eq. 4 , from which we obtained: $k_{m} \approx 2.2 / R_{2}$ (continuous line) and $R_{c} \approx 0.019$ (dashed line).

Finally, we try to evaluate the typical size $S$ of the clusters which appear in the spiky phase. The typical cluster size was calculated averaging the root mean square dispersion for all the clusters present in the system at a given time. Data exposed in Figure 7 show a dependence of the 
cluster size on the diffusion coefficient: $S \propto \sigma$, equivalent to $S \propto \sqrt{D}$. We can easily interpret this result. Since there is no attraction or repulsion between individuals, they will experience just a diffusive motion during their lifetimes. Assuming that the individuals confined in a cluster diffuse a distance proportional to $\sqrt{D}$, the dependence of the clusters size on the diffusion coefficient shown in Figure 7 is straightforward. Similar results were reported for simpler models in $[23,25]$.

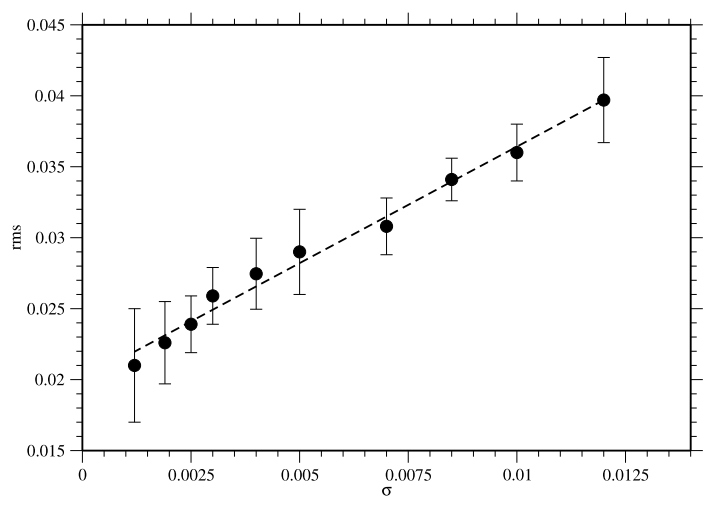

FIG. 7. Cluster size as a function of $\sigma$. The dashed line has slope 1, which corresponds to a square root dependence of the cluster size on the diffusion coefficient.

\section{CONCLUSIONS}

We have developed an in-deep analysis of a spatial Lotka-Volterra model characterised by a finite range predator-prey interaction in a two dimensional space. This physical space is obviously the most appropriate for describing real ecosystems.

This model can be considered relevant from an ecological perspective for two reasons. First, because it contributes to the discussion about the mechanisms that can generate spatial correlations in population ecology, showing how preys and predators interaction can be considered among them. This conjecture is of particular importance as some records of spatial correlations between preys and predators were clearly reported $[2,3]$. Second, because the emergence of spatial patterns in an erratic oscillatory regime, which can contemplate predators extinction, shows realistic elements generally absent from conventional descriptions.

A theoretical interest related to this kind of studies exists too. From this perspective, we demonstrate that in this model instability is driven by the form of the interaction and is independent of the diffusion process. Moreover, we carried out our analysis developing a direct comparison between an individual based implementation and a mean-field description, showing a perfect match between them for many quantitative features, a fact that is not always achieved $[5,11,23,26]$. Finally, we show how the presence of structures persists independently of the space dimension where the model can be implemented.

[1] P.A.P. Moran, Aust. J. Zool. 1, 291 (1953).

[2] A. Liebhold, W. D. Koenig, and O. N. Bjrnstad, Annu. Rev. Ecol. Evol. Syst. 35, 467,(2004).

[3] P.C. Tobin and O.N. Bjørnstad, J. Anim. Ecol., 72, 460 (2003).

[4] T. Biancalani and D. Fanelli, Phys. Rev. E 81, 046215, (2010).

[5] T. Butler and N. Goldenfeld, Phys. Rev. E 80030902 (2009).

[6] M. Scott, F. J. Poulin and H. Tang, Proc. R. Soc. A, (2010).

[7] J. Bascompte and R.V. Solé, Trends Ecol. Evol., 10, 361, (1995); O.N. Bjørnstad, R.A. Ims and X. Lambin, Trends Ecol. Evol., 14, 427, (1999).

[8] Hebblewhite, E. H. Merrill and T. L. McDonald, Oikos, 111, (2005).

[9] A. M. Turing, Phil. Trans. R. Soc. Lond. B, 237, (1953).

[10] J.D. Murray, Mathematical Biology, Springer (1989).

[11] C. A. Lugo and Alan J. Mckane., Phys. Rev. E 78, 051911 (2008).

[12] S. L. Lima, Trends Ecol. Evol. 17, 70, (2002).

[13] E. Brigatti, M. Oliva, M. Núñez-López, R. OliverosRamos, J. Benavides, Europhys. Lett., 88, 68002, (2009).

[14] E.E. Holmes, M.A. Lewis, J.E. Banks and R.R.Veit, Ecology 75, 17 (1994); D. Alonso, F. Bartumeus and J. Catalan, Ecology, 83, 28, (2002).

[15] R. MacArthur, and R. Levins, Am. Nat., 101, 377 (1967); J. Roughgarden, Theory of Population Genetics and Evolutionary Ecology: an Introduction. Macmillan Publishers (1979); M. A. Fuentes, M. N. Kuperman, and V. M. Kenkre, Phys. Rev. Lett. 91, 158104 (2003); P. Szabó and G. Meszéna, Oikos 112, 612 (2006); M. Scheffer, E.H. Van Nes, Proc. Natl. Acad. Sci. USA, 103, 6230 (2006).

[16] F. Bagnoli and M. Bezzi, Phys. Rev. Lett. 793302 (1997); U. Dieckmann and M. Doebeli, Nature 400, 354 (1999); V. Schwämmle and E. Brigatti, Europhys. Lett. 75, 342 (2006); E. Brigatti, J. S. Sá Martins and I. Roditi, Physica A 376, 378 (2007).

[17] E. Hernandez-Garcia and C. Lopez, Phys. Rev. E 70, 016216 (2004).

[18] M. Mobilia, I. T. Georgiev and U. C. Tauber, J. Stat. Phys., 128, 447 (2007).

[19] Anna et al., Phys. Rev E, 81, 056110 (2010)

[20] B. Houchmandzadeh, Phys. Rev. E 66, 052902 (2002).

[21] D. Mollison, Mathematical Biosciences 107, 255 (1991).

[22] W.G. Wilson, A.M. De Roos, and E. McCauley, Theoretical Population Biology, 43, 91 (1993); J. E. Satulovsky and T. Tomé, Phys. Rev. E 49, 5073 (1994); A. Lipowski, Phys. Rev. E 60, 5179 (1999).

[23] E. Brigatti,V. Schwämmle, and M.A. Neto, Phys. Rev. 
E 77, 021914 (2008).

[24] Dauxois et al. Phys. Rev E 79, 036112 (2009); Bladon et al. Phys. Rev. E 81, 066121 (2010); Black et al., Phys. Rev E 80, 021922 (2009).
25] E. Hernandez-Garcia and C. Lopez, Physica A 356, 95 (2005).

[26] A. J. McKane and T. J. Newman, Phys. Rev. Lett. 94, 218102 (2005). 